\title{
Mixed Tumor of the Parotid Gland
}

National Cancer Institute

\section{Source}

National Cancer Institute. Mixed Tumor of the Parotid Gland. NCI Thesaurus. Code C35416.

A benign or malignant neoplasm that arises from the parotid gland. It is characterized by the presence of epithelial and mesenchymal elements. This category includes pleomorphic adenoma, carcinoma ex pleomorphic adenoma, and carcinosarcoma. 